\title{
Phase evolution of terahertz radiation from femtosecond laser-induced air plasma
}

\author{
Zhen Zhang, ${ }^{1}$ YAnping Chen, ${ }^{1,2,}{ }^{*}$ Zhelin Zhang, $1,2,4$ TIAnhaO XiA, 1,2 \\ JiAYANG ZHANG, ${ }^{1,2}$ Zhengming Sheng, ${ }^{1,2,3,4}$ AND JIE ZHANG ${ }^{1,2}$ \\ ${ }^{1}$ Key Laboratory for Laser Plasma (Ministry of Education), School of Physics and Astronomy, Shanghai Jiao Tong University, Shanghai 200240, China \\ ${ }^{2}$ IFSA Collaborative Innovation Center, Shanghai Jiao Tong University, Shanghai 200240, China \\ ${ }^{3}$ Department of Physics, SUPA, University of Strathclyde, Glasgow G4 ONG, UK \\ ${ }^{4}$ Tsung-Dao Lee Institute, Shanghai, China. \\ *Corresponding author: yanping.chen@sjtu.edu.cn
}

\begin{abstract}
The phase evolution of terahertz radiation from singlecolor femotsecond laser induced air plasma controlled by a DC-bias is investigated experimentally. When the DCbias is moved from the end to the beginning of the laserplasma filament, the produced terahertz waveform is advanced temporally and its carrier-envelope phase changed. Our phase spectrum analysis suggests that the slope and the intercept of the phase spectrum determine respectively the temporal shift and the carrier-envelope phase of the terahertz waveform. Therefore, the observed terahertz waveform evolution is mainly due to the $\mathrm{THz}$ propagation effect in plasma filament and the Gouy phase shift associated with the detection scheme. Our work also illustrates explicitly the temporal order of terahertz radiation from different parts of a filament.
\end{abstract}

Femtosecond laser-induced air plasma can radiate terahertz $(\mathrm{THz})$ pulses with high field strength [1] and broad spectral bandwidth [2]. $\mathrm{THz}$ waves with high electric fields can be used to study charge carrier dynamics in semiconductors [3], transient photoconductivity in GaAs [4] and impact ionization dynamics[5]. THz spectrum with large bandwidth is beneficial for $\mathrm{THz}$ wave remote sensing and spectroscopy [6]. Compared with the terahertz radiation from a laserirradiated solid target, $\mathrm{THz}$ wave generation in air plasma can be driven by intense femtosecond laser pulses with moderate peak power and focused intensity operating at $\mathrm{kHz}$ high repetition rates [7]. As the $\mathrm{THz}$ pulses produced in this way are usually with few cycles, understanding their phase evolution under different experimental conditions is beneficial for their applications.

A lot of research work has been carried out on the subject of $\mathrm{THz}$ waveform evolution. Kim et al. showed the change of the temporal $\mathrm{THz}$ waveform by controlling the relative phase between fundamental and second harmonic pulses [8]. Bai et al. derived the evolution of $\mathrm{THz}$ waveforms driven by few-cycle laser pulses from the shift of the carrier phase and the change of the laser envelope during propagation [9]. Zhao et al. investigated the propagation effect of $\mathrm{THz}$ waves inside the filaments, which is responsible for the significant advancement of the maximum value of the $\mathrm{THz}$ waveform generated by the femtosecond laser filament in the air [10]. In the study of $\mathrm{THz}$ wave generation by two-color lasers in nitrogen molecules from the supersonic gas nozzle, Li et al. revealed that the shift of the waveform was due to the change in the refractive index of the THz wave [11]. In 2018, Li et al. confirmed that the competition effect of laser plasma absorption and oscillation currents could determine the evolution of the THz pulses [12]. Zhang et al. proposed that the carrier-envelope phase of the $\mathrm{THz}$ pulse could be controlled by varying the filament length and the initial phase difference between the two-color laser components [13]. Wang et al. have reported that the $\mathrm{THz}$ waveform could have a phase shift of $\pi$ due to the change of the plasma density distribution at the beginning and end of a laser filament [14].

As one's understanding of the evolution of the $\mathrm{THz}$ waveform becomes deeper, it is gradually recognized that $\mathrm{THz}$ radiation is a superposition of $\mathrm{THz}$ waves radiated from different positions of the filament. Panov et al. reported the role of constructive interference in the determination of far-field $\mathrm{THz}$ profile [15]. You et al. demonstrated that the far-field $\mathrm{THz}$ radiation profiles are due to offaxis constructive interference between locally generated $\mathrm{THz}$ waves along the filament [16]. Zhang et al. proposed a radiation model, which suggested that $\mathrm{THz}$ radiation from a filament was due to coherently superposition of $\mathrm{THz}$ pulses from a linear array of dipoles along the filament [17]. As such, the $\mathrm{THz}$ waveform or carrierenvelope phase is closely associated with the temporal order of $\mathrm{THz}$ waves radiated from different parts of a long filament, which is still not all clear.

In this Letter, the waveform evolution of $\mathrm{THz}$ radiation from a single-color air filament is investigated by changing the position of a slice-shaped external electric field (DC-biased field) applied on the filament. A temporal shift, as well as a change in carrier-envelope phase of the measured $\mathrm{THz}$ waveform, can be explained by phase spectral modification due to the $\mathrm{THz}$ pulse propagation effect. Our finding is helpful for understanding the physical mechanisms of $\mathrm{THz}$ wave generation from femtosecond laser-induced air plasma.

The experimental setup is shown in Fig. 1, where a $1 \mathrm{kHz}, 40 \mathrm{fs}$, $800 \mathrm{~nm}, 5 \mathrm{~mJ}$ laser pulse is focused by a convex lens with a focal length of $30 \mathrm{~cm}$, forming a centimeter-long filament in air. A sliceshaped external DC electric field of $8 \mathrm{kV} / \mathrm{cm}$, induced by two pieces of 3-mm-wide copper strips, was applied perpendicularly on the filament. The copper strips were placed on a motive stage so that it can be translated along the filament. Since the amplitude of the $\mathrm{THz}$ pulse from $8 \mathrm{kV} / \mathrm{cm}$ DC-biased plasma is about two orders of magnitude higher than that from single-color plasma [18], the measured $\mathrm{THz}$ signal in our setup is mainly contributed by $\mathrm{THz}$ radiation from plasma zone which is affected by the DC-bias. By scanning the slice-shaped external electric field, $\mathrm{THz}$ waves from different parts (DC-biased) of the filament can be collected by two off-axis parabolic mirrors and measured by the electro-optic sampling (EOS) method. We didn't shift the EO crystal while the DC-bias was moved. We optimized the position of the EO crystal when the sliceshaped DC-bias was fixed at $\mathrm{z}=6 \mathrm{~mm}$. The diameter of the off-axis parabolic mirror is 2 inches, and the focal length of the two off-axis parabolic mirrors is about $100 \mathrm{~mm}$. The EO crystal is oriented in $\langle 110\rangle$, and the thickness of the crystal is about $1.5 \mathrm{~mm}$. The optical 
axis of the EO crystal is parallel to the direction of the DC-biased field.

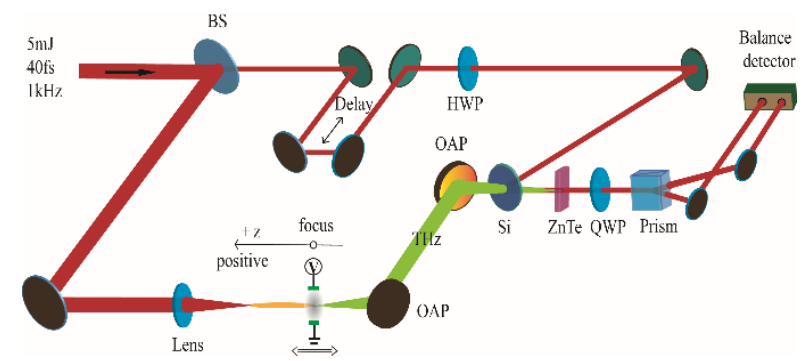

Fig. 1. (Color online) Schematic diagram of the experimental setup. The distance between the center of the slice-shaped DC-bias and the geometric focus of the lens (or the end of the filament) is defined as z. BS: beam splitter; OAP: off-axis parabolic; HWP: half-wave plate; QWP: quarter-wave plate.

We measured the waveforms of the $\mathrm{THz}$ radiation from DC-biased air filament when the slice-shaped DC-bias was scanned from $0 \mathrm{~mm}$ to $15 \mathrm{~mm}$ from the geometric focus of the lens ( $z=0 \mathrm{~mm}$ to $\mathrm{z}=15 \mathrm{~mm}$ ), as shown in Fig. 2(a).

One can see that the $\mathrm{THz}$ waveform changes slightly as $z$ increases. The THz waveforms shown in Fig. 2(a) are not normalized. And the $\mathrm{THz}$ amplitude remains substantially unchanged as the electrode moves. Meanwhile, it shows that the peak of the $\mathrm{THz}$ waveform advances temporally. Figure 2(b) shows the spectra of the THz waves from Fig. 2(a) at several typical locations. We can find that the peak frequency and bandwidth of the $\mathrm{THz}$ wave remain almost unchanged. In order to explore the physical mechanism of this phenomena, we introduce the phase spectrum analysis of $\mathrm{THz}$ radiation.
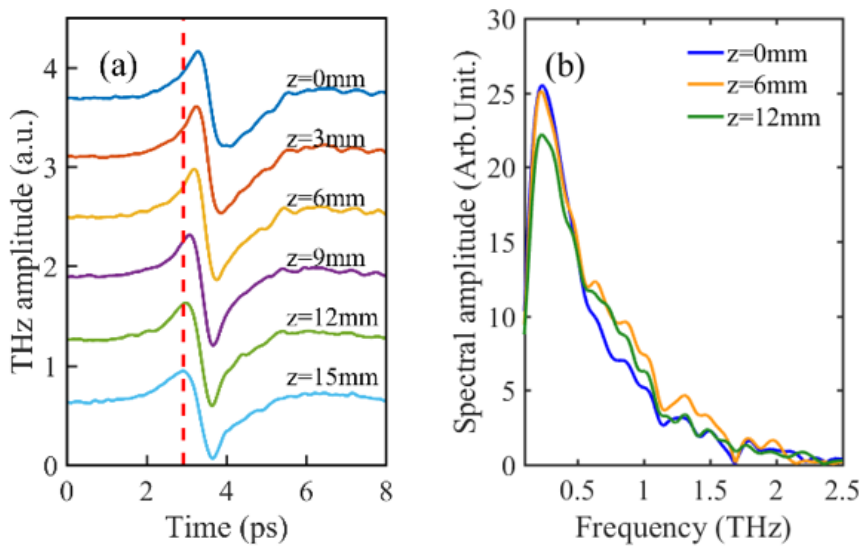

Fig. 2. (a) Measured waveforms of the THz radiation when the sliceshaped DC-bias located at $z=0,3,6,9,12$ and $15 \mathrm{~mm}$. The pump laser energy is $5 \mathrm{~mJ}$. A red dash line is added to help identify the shift of the peak of the waveform. (b) Typical spectra of the THz waveforms at three positions of the DC-biased field.

For a given $\mathrm{THz}$ waveform, we can obtain both its amplitude spectrum and phase information using Fourier Transform method, as shown in Fig. 3. Figures 3(a) and 3(c) show typical THz waveforms when slice-shaped DC-biased field is applied respectively at the end and the beginning of the filament, while Figs. 3(b) and 3(d) are their corresponding Fourier transformed spectra (blue curve) and phases (black curve), respectively. It is found that the phase $\varphi$ has an almost linear relationship with the $\mathrm{THz}$ frequency, so it can be expressed by

$$
\varphi=\alpha \cdot \Omega+\beta,
$$

where $\alpha$ and $\beta$ are the slope and the intercept of the phase spectrum, $\Omega$ is the angular frequency of the $\mathrm{THz}$ waves, as shown in Figs. 3(b) and 3(d) (red dashed lines). When the slice-shaped DCbiased field is applied at the end of the filament $(z=0 \mathrm{~mm})$, we have the fitted values $\alpha=-3.56 \mathrm{ps}$ and $\beta=0.33 \pi$ for the THz phase spectra. Here, $\alpha=-3.56$ ps means that a probe pulse arrives 3.56 ps ahead of the THz pulse when measuring the waveform of this $\mathrm{THz}$ pulse by the electro-optic sampling method. When the slice-shaped DC-biased field is applied at the beginning of the filament $(z=15 \mathrm{~mm})$, we have $\alpha=-3.46 \mathrm{ps}$ and $\beta=0.58 \pi$. When the THz waveform is different, the phase spectra are different. When the difference between the amplitude spectrum of the $\mathrm{THz}$ wave is small, the temporal waveform of the $\mathrm{THz}$ wave is mainly determined by the slope and intercept of the phase spectrum. Therefore, we can understand the evolution of the $\mathrm{THz}$ waveform by analyzing the changes in the phase spectrum.
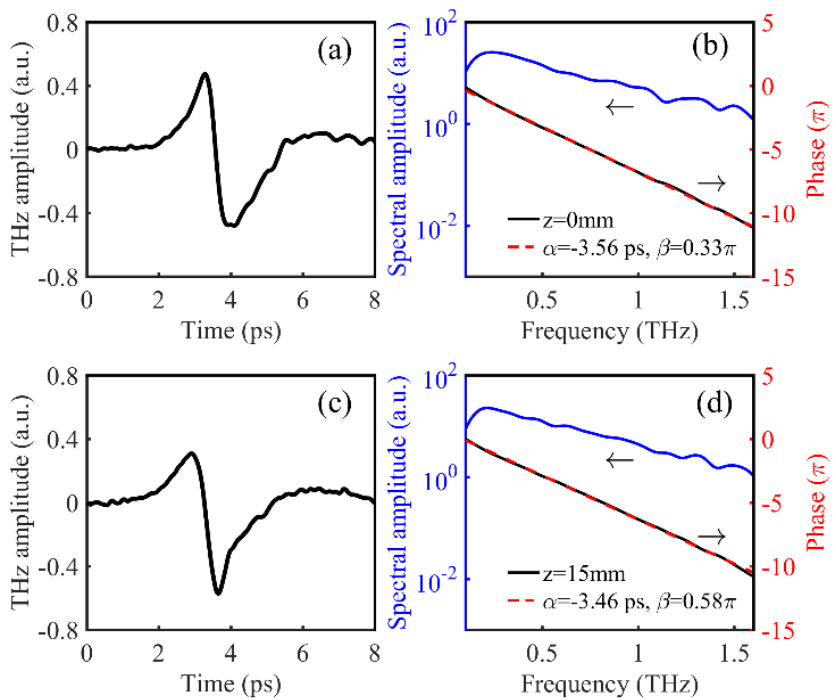

Fig. 3. (a) and (c) show THz waveforms when the DC-bias is applied at $z=0 \mathrm{~mm}$ and $z=15 \mathrm{~mm}$. The blue curves in (b) and (d) are the spectra, and black curves in (d) and (d) are the phases. The red dashed line in (b) and (d) are the fitted curves of the phase spectrum, respectively.

In the following, we will clarify the relation between the phase spectra and the corresponding $\mathrm{THz}$ waveforms. Assume we have the time domain signal $x(t)$ and its corresponding complex amplitude $X(\Omega)$ in the frequency domain. The relation between $x(t)$ and $X(\Omega)$ satisfies [19]:

$$
x(t)=\frac{1}{2 \pi} \int_{-\infty}^{\infty} X(\Omega) \mathrm{e}^{\mathrm{i} \Omega t} \mathrm{~d} \Omega,
$$

In the frequency domain, as the phase changes by $\Delta \varphi=\Delta \alpha \cdot \Omega+\Delta \beta$, the new signal in the time domain $x_{\mathrm{n}}(t)$ can be written as:

$$
x_{\mathrm{n}}(t)=\frac{1}{2 \pi} \int_{-\infty}^{\infty} X(\Omega) \mathrm{e}^{\mathrm{i} \Omega t} \cdot \mathrm{e}^{\mathrm{i}(\Delta \alpha \cdot \Omega+\Delta \beta)} \mathrm{d} \Omega,
$$

After simplification, we can get: 


$$
x_{\mathrm{n}}(t)=x(t+\Delta \alpha) \mathrm{e}^{\mathrm{i} \Delta \beta} .
$$

This means that the carrier-envelope phase of the time domain signal changes by $\Delta \beta$ when the intercept of the phase spectrum changes $\Delta \beta$, as shown in Figs. 4(a) and 4(b) ( $\Delta \alpha=0$ and $\Delta \beta=\pi / 2$ ). When the slope of the phase spectrum changes by $\Delta \alpha$, the time domain signal shifts $\Delta \alpha$ in time, as shown in Figs. 4(c) and 4(d) ( $\Delta \alpha=0.26 \mathrm{ps}$ and $\Delta \beta=0$ ). Therefore, modification of a $\mathrm{THz}$ waveform (including time shift and carrier-envelope phase change) could be contributed by the change of the slope ( $\Delta \alpha)$ and the intercept ( $\Delta \beta$ ) in its phase spectrum.
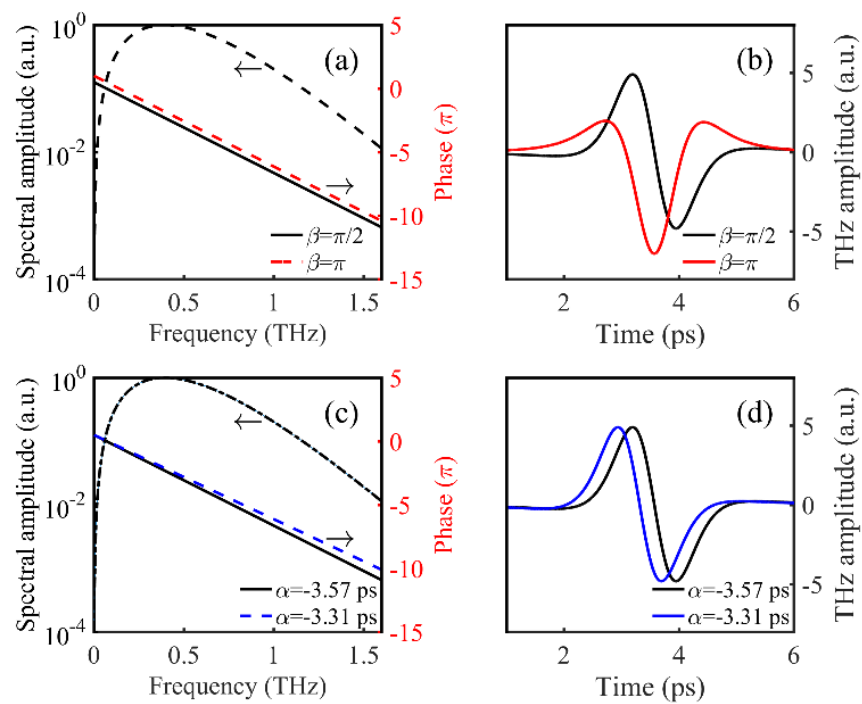

Fig. 4. (a) The amplitude spectrum (short dashed line) and two phase spectra with different intercepts ( $\beta=\pi / 2$ for solid line and $\beta=\pi$ for dashed line) when the slope of the phase spectra is $-3.57 \mathrm{ps}$. Their corresponding $\mathrm{THz}$ waveforms are shown in (b). (c) The amplitude spectrum (dot dashed line) and two phase spectra with different slopes ( $\alpha=-3.57 \mathrm{ps}$ for solid line and $\alpha=-3.31 \mathrm{ps}$ for dashed line) when the intercept of the phase spectra is $\pi / 2$. Their corresponding $\mathrm{THz}$ waveforms are shown in (d).

In our experiment, the measured $\mathrm{THz}$ signal in the far field [as Fig. 5 (a)] is a coherent superposition of $\mathrm{THz}$ radiation emitted from different point sources along the filament, which is mainly contributed by $\mathrm{THz}$ radiation from plasma zone with a DC-bias. As this DC-bias is located at $Z$, the phase of the emitted THz wave in the frequency domain can be written as:

$$
\varphi=\varphi_{0}-\varphi_{1}(z)-\frac{\Omega}{c} l(z),
$$

where $c$ is the velocity of light, $l(z)$ is the propagation distance from the source to the detector in the far field. In Eq. (5), the first part, $\varphi_{0}$, is the initial carrier-envelope phase of the THz radiation, which keeps constant for any point source along the filament. The second part of Eq. (5), $\varphi_{1}(z)$, could be written as $\varphi_{1}(z)=\frac{\Omega}{c} z \frac{v^{800}}{v^{T H z}}+\phi_{G}$, where $v^{T H z}$ is the velocity of the THz pulse and $v^{800}$ is the velocity of the optical pump. The first term of $\varphi_{1}(z)$ comes from the velocity mismatch and the second term of $\varphi_{1}(z)$ comes from the
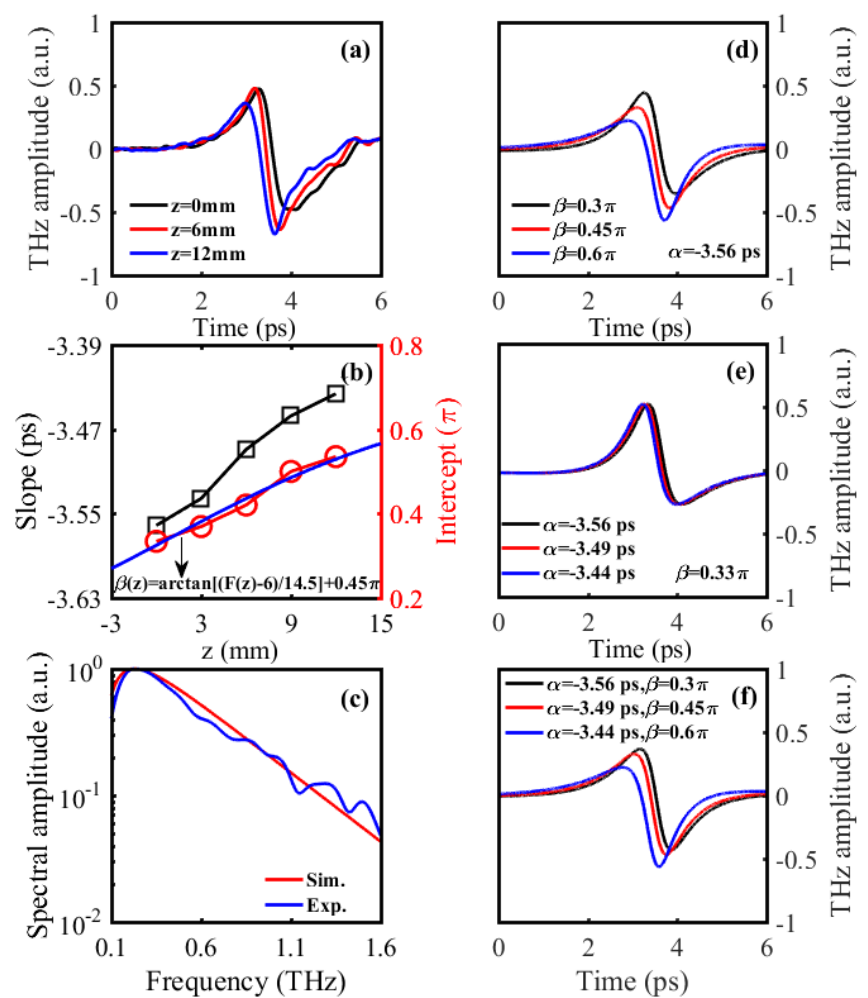

Fig. 5. (a) Measured $\mathrm{THz}$ waveform from air plasmas with sliceshaped DC-bias at $z=0,6,12 \mathrm{~mm}$. (b) The slope (black rectangles) and the intercept (red circles) of the $\mathrm{THz}$ phase spectrum versus different DC-bias positions. (c) Typical THz spectra, with the blue curve from measurement and red curve for simulation. The retrieved $\mathrm{THz}$ waveforms from simulation as a function of the intercept of the phase spectrum only (d), slope of the phase spectrum only (e), and both of the intercept and the slope of the phase spectrum (f), respectively.

contribution of the Gouy phase shift [20]. The velocity mismatch mainly affects the slope of the $\mathrm{THz}$ phase spectrum and the Gouy phase shift mainly affects the intercept of the $\mathrm{THz}$ phase spectrum. In case of $l(z)=0$, for example, when there is no velocity mismatch between the optical pump and the THz pulse, the slope of the phase spectrum will not change, i.e., no time shift of the THz pulse. When velocity mismatch between the optical pump and the THz pulse occurs, it results in an increase in the slope of the $\mathrm{THz}$ phase spectrum, i.e., a temporal advance of the THz pulse, as shown in Fig. 4(c) and Fig. 4(d). We can fit the experimental data as $\varphi=\alpha(z) \Omega+\beta(z)$. If $\alpha(z)$ and $\beta(z)$ are determined, however, it is still impossible to estimate the value of $v^{800} / v^{T H z}$ due to the complexity of $l(z)$. Figure 5(b) shows the intercepts of the phase spectra (see red circles) of $\mathrm{THz}$ waves measured when the slice-shaped DC-bias is located at different positions. This tells that the intercept of the phase spectrum obeys the relation of the Gouy phase shift $\phi_{G}\left(z^{\prime}\right)=-\arctan \left(z^{\prime} / z_{R}\right)$

when $Z^{\prime}$ changes within a small range [21], $Z^{\prime}$ is the distance from the THz focus to the EO crystal. Here $Z_{R}$ is the Rayleigh length of the $\mathrm{THz}$ pulse. In our case, the diameter of the $\mathrm{THz}$ beam irradiated on the off-axis parabolic mirror is $\mathrm{D}=32.4 \mathrm{~mm}$ and the focal length of the off-axis parabolic mirror is $\mathrm{f}=100 \mathrm{~mm}$, the peak frequency of the $\mathrm{THz}$ wave is about $0.23 \mathrm{THz}$, one can estimate the $\mathrm{THz}$ beam waist after the parabolic mirror $w_{0}=2.5 \mathrm{~mm}$ and the corresponding 
Rayleigh length $z_{R}=14.5 \mathrm{~mm}$. With this, we can fit the intercept well with $\beta(\mathrm{z})=\varphi_{0}+\arctan \left[\frac{\mathrm{F}(\mathrm{z})-6}{\mathrm{z}_{R}}\right]$ according to the Eq. (5) (see blue curve in Fig. 5(b)), where $\mathrm{F}(z)=f-1 /[0.02-1 /(z+f)]$ and $\varphi_{0}=0.45 \pi$. And its influence on the waveform of the $\mathrm{THz}$ signal is shown in Fig. 5(d). The third part of Eq.(5), $\frac{\Omega}{C} l(z)$, is the phase variation due to the propagation effect of the THz wave when it propagates from the source to the detector in far field. It also affects the slope of the $\mathrm{THz}$ phase spectrum. The measured slopes of the $\mathrm{THz}$ phase spectra (black rectangles) versus the DC-bias position is shown in Fig. 5(b). Its corresponding effect on the THz waveform is shown in Fig. 5(e). When considering the velocity mismatch between the optical pump and the THz pulse, the THz propagation effect and the Gouy phase shift, we can retrieve the $\mathrm{THz}$ waveforms radiated from air plasmas with slice-shaped DC-bias at $z=0,6,12 \mathrm{~mm}$ [Fig. 5(f)], which well agrees with the measured $\mathrm{THz}$ waveforms in Fig. 5(a).

The slope of the phase spectrum or the $\mathrm{THz}$ pulse delay is also related to the pump laser energy in addition to the DC-bias position. Figure 6 shows that the slopes of the $\mathrm{THz}$ phase spectrum increase when the laser energy increases. This suggests a temporal advance of the emitted $\mathrm{THz}$ pulse with an increase in laser energy. Therefore, higher laser energy creates a longer laser plasma filament, and thus leads to faster propagation of the emitted $\mathrm{THz}$ pulse, in agreement with our previous observation [17].

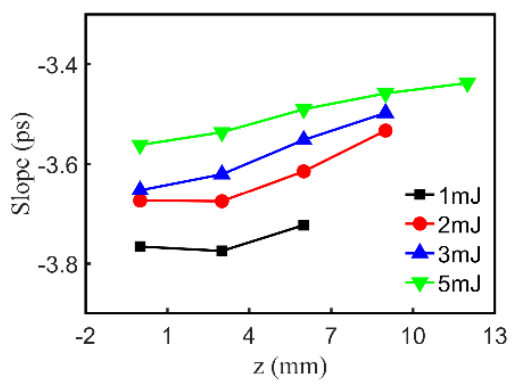

Fig. 6. Relationship between the slope of the THz phase spectrum and DC-bias position $\mathrm{z}$ for different laser energies.

In summary, we have investigated the phase evolution of $\mathrm{THz}$ waveforms from femtosecond laser-induced air plasma by controlling the DC-bias position along the filament. It is found that the variation of the $\mathrm{THz}$ waveforms is caused by the combined effects of the temporal shift of the $\mathrm{THz}$ waveform and the changes of the carrierenvelope phase of the THz pulse when the DC-bias position is moved. The slope of the phase spectrum determines the shift of the $\mathrm{THz}$ waveforms temporally and the intercept of the phase spectrum determines the changes of the carrier-envelope phases of the $\mathrm{THz}$ pulses. As the position of the applied DC-biased field moves to the beginning of the laser filament, the intercept of the phase spectrum or the carrier-envelope-phase changes, which is related to the Gouy phase shift. The velocity mismatch between the $\mathrm{THz}$ pulse and the optical pump, together with the $\mathrm{THz}$ propagation effect between the point source and the detector in far field, leads to an increase in the slope of the $\mathrm{THz}$ phase spectrum or a temporal shift of the THz pulse. These effects also exist when using a two-color laser scheme for $\mathrm{THz}$ radiation. In such case, the phase evolution of the $\mathrm{THz}$ pulse is dominated not only by combined effects mentioned above but also the phase difference between the two-color laser components. By clarifying the temporal order of the $\mathrm{THz}$ wave generation at different positions of the filament, the general understanding of the generation mechanism of the THz radiation from the femtosecond laser-induced air plasma can be enhanced.

Funding. The National Natural Science Foundation of China (Grant No. 11774228, 11655002, 11721091, 11474202 and 11991070) and Science and Technology Commission of Shanghai Municipality (Grant No. 16DZ2260200).

Disclosures: The authors declare no conflicts of interest.

\section{REFERENCES}

1. T. Bartel, P. Gaal, K. Reimann, M. Woerner, and T. Elsaesser, Opt. Lett 30, 2805-2807 (2005).

2. N. Karpowicz, J. Dai, X. Lu, Y. Chen, M. Yamaguchi, H. Zhao, X.-C. Zhang, L. Zhang, C. Zhang, and M. PriceGallagher, Appl. Phys. Lett 92, 011131 (2008).

3. R. Ulbricht, E. Hendry, J. Shan, T. F. Heinz, and M. Bonn, Rev. Mod. Phys. 83, 543 (2011).

4. M. C. Beard, G. M. Turner, and C. A. Schmuttenmaer, Phys. Rev. B 62, 15764 (2000).

5. M. C. Hoffmann, J. Hebling, H. Y. Hwang, K.-L. Yeh, and K. A. Nelson, Phys. Rev. B 79, 161201 (2009).

6. J. Dai, X. Xie, and X.-C. Zhang, Phys. Rev. Lett 97, 103903 (2006).

7. K. Mori, M. Hashida, T. Nagashima, D. Li, K. Teramoto, Y. Nakamiya, S. Inoue, and S. Sakabe, AIP Advances 9, 015134 (2019).

8. K.-Y. Kim, J. H. Glownia, A. J. Taylor, and G. Rodriguez, Opt. Express 15, 4577-4584 (2007).

9. Y. Bai, L. Song, R. Xu, C. Li, P. Liu, Z. Zeng, Z. Zhang, H. Lu, R. Li, and Z. Xu, Phys. Rev. Lett 108, 255004 (2012).

10. J. Zhao, Y. Zhang, Z. Wang, W. Chu, B. Zeng, W. Liu, Y. Cheng, and Z. Xu, Laser Phys. Lett 11, 095302 (2014).

11. N. Li, Y. Bai, T. Miao, P. Liu, R. Li, and Z. Xu, Opt. Express 24, 23009-23017 (2016).

12. X. Li, Y. Bai, N. Li, and P. Liu, Opt. Lett 43, 114-117 (2018).

13. Z. Zhang, Y. Chen, M. Chen, Z. Zhang, J. Yu, Z. Sheng, and J. Zhang, Phys. Rev. Lett 117, 243901 (2016).

14. T.-J. Wang, J. Ju, Y. Liu, R. Li, Z. Xu, and S. Leang Chin, Appl. Phys. Lett 110, 221102 (2017).

15. N. Panov, O. Kosareva, V. Andreeva, A. Savel'ev, D. Uryupina, R. volkov, V. Makarov, A. Shkurinov, JETP Lett 93, 638(2011).

16. Y. You, T. Oh, and K. Kim, Phys. Rev. Lett 109, 183902 (2012).

17. Z. Zhang, Y. Chen, S. Cui, F. He, M. Chen, Z. Zhang, J. Yu, L. Chen, Z. Sheng, and J. Zhang, Nature Photonics 12, 554 (2018).

18. Y. Chen, T.-j. Wang, C. Marceau, F. Théberge, M. Châteauneuf, J. Dubois, O. Kosareva, and S. L. Chin, Appl. Phys. Lett. 95, 101101 (2009).

19. J. R. Fienup, Opt. Lett 3, 27-29 (1978).

20. S. Feng and H. G. Winful, Opt. Lett 26, 485-487(2001).

21. A. B. Ruffin, J.V. Rudd, J.F. Whitaker, S. Feng, and H.G. Winful, Phys. Rev. Lett. 83, 3410-3413(1999). 


\section{REFERENCES}

1.T. Bartel, P. Gaal, K. Reimann, M. Woerner, and T. Elsaesser, "Generation of single-cycle $\mathrm{THz}$ transients with high electric-field amplitudes," Optics Letters 30, 2805-2807 (2005).

2. N. Karpowicz, J. Dai, X. Lu, Y. Chen, M. Yamaguchi, H. Zhao, X.-C. Zhang, L. Zhang, C. Zhang, and M. PriceGallagher, "Coherent heterodyne time-domain spectrometry covering the entire "terahertz gap"," Applied Physics Letters 92, 011131 (2008).

3. R. Ulbricht, E. Hendry, J. Shan, T. F. Heinz, and M. Bonn, "Carrier dynamics in semiconductors studied with timeresolved terahertz spectroscopy," Reviews of Modern Physics 83, 543 (2011).

4. M. C. Beard, G. M. Turner, and C. A. Schmuttenmaer, "Transient photoconductivity in GaAs as measured by timeresolved terahertz spectroscopy," Physical Review B 62, 15764 (2000).

5. M. C. Hoffmann, J. Hebling, H. Y. Hwang, K.-L. Yeh, and K. A. Nelson, "Impact ionization in InSb probed by terahertz pump-terahertz probe spectroscopy," Physical Review B 79, 161201 (2009).

6. J. Dai, X. Xie, and X.-C. Zhang, "Detection of broadband terahertz waves with a laser-induced plasma in gases," Physical Review Letters 97, 103903 (2006).

7. K. Mori, M. Hashida, T. Nagashima, D. Li, K. Teramoto, Y. Nakamiya, S. Inoue, and S. Sakabe, "Increased energy of $\mathrm{THz}$ waves from a cluster plasma by optimizing laser pulse duration," AIP Advances 9, 015134 (2019).

8. K.-Y. Kim, J. H. Glownia, A. J. Taylor, and G. Rodriguez, "Terahertz emission from ultrafast ionizing air in symmetrybroken laser fields," Optics Express 15, 4577-4584 (2007).

9. Y. Bai, L. Song, R. Xu, C. Li, P. Liu, Z. Zeng, Z. Zhang, H. $\mathrm{Lu}, \mathrm{R}$. Li, and $\mathrm{Z}$. Xu, "Waveform-controlled terahertz radiation from the air filament produced by few-cycle laser pulses," Physical Review Letters 108, 255004 (2012).

10. J. Zhao, Y. Zhang, Z. Wang, W. Chu, B. Zeng, W. Liu, Y. Cheng, and $\mathrm{Z}$. $\mathrm{Xu}$, "Propagation of terahertz wave inside femtosecond laser filament in air," Laser Physics Letters 11, 095302 (2014).

11. N. Li, Y. Bai, T. Miao, P. Liu, R. Li, and Z. Xu, "Revealing plasma oscillation in THz spectrum from laser plasma of molecular jet," Optics Express 24, 23009-23017 (2016).

12. X. Li, Y. Bai, N. Li, and P. Liu, "Absorption of laser plasma in competition with oscillation currents for a terahertz spectrum," Optics Letters 43, 114-117 (2018).

13. Z. Zhang, Y. Chen, M. Chen, Z. Zhang, J. Yu, Z. Sheng, and J. Zhang, "Controllable terahertz radiation from a lineardipole array formed by a two-color laser filament in air," Physical Review Letters 117, 243901 (2016).

14. T.-J. Wang, J. Ju, Y. Liu, R. Li, Z. Xu, and S. Leang Chin, "Waveform control of enhanced $\mathrm{THz}$ radiation from femtosecond laser filament in air," Applied Physics Letters 110, 221102 (2017).
15. N. Panov, O. Kosareva, V. Andreeva, A. Savel'ev, D. Uryupina, R. volkov, V. Makarov, A. Shkurinov, JETP Letters 93, 638(2011).

16. Y. You, T. Oh, and K. Kim, "Off-axis phase-matched terahertz emission from two-color laser-induced plasma filaments," Physical Review Letters 109, 183902 (2012).

17. Z. Zhang, Y. Chen, S. Cui, F. He, M. Chen, Z. Zhang, J. Yu, L. Chen, Z. Sheng, and J. Zhang, "Manipulation of polarizations for broadband terahertz waves emitted from laser plasma filaments," Nature Photonics 12, 554 (2018).

18. Y. Chen, T.-j. Wang, C. Marceau, F. Théberge, M. Châteauneuf, J. Dubois, O. Kosareva, and S. L. Chin, "Characterization of terahertz emission from a dc-biased filament in air," Applied Physics Letters 95, 101101 (2009).

19. J. R. Fienup, "Reconstruction of an object from the modulus of its Fourier transform," Optics Letters 3, 27-29 (1978).

20. S. Feng and H. G. Winful, "Physical origin of the Gouy phase shift", Optics Letters 26, 485-487(2001).

21. A. B. Ruffin, J.V. Rudd, J.F. Whitaker, S. Feng, and H.G. Winful, "Direct observation of the Gouy phase shift with single-cycle terahertz pulses",Physics Review Letters 83, 3410-3413(1999). 